\title{
Indicadores para a ocorrência da dengue em municípios do oeste de Santa Catarina,
}

\section{Brasil}

\author{
Indicators to dengue occurrence in municipalities of western Santa Catarina, Brazil \\ Indicadores para la ocurrencia de dengue en municipios del oeste de Santa Catarina, Brasil
}

Recebido: 20/07/2021 | Revisado: 27/07/2021 | Aceito: 28/07/2021 | Publicado: 04/08/2021

Joel Morschbacher

ORCID: https://orcid.org/0000-0003-1755-8783 Universidade Comunitária da Região de Chapecó, Brasil E-mail: joel.m@unochapeco.edu.br

Lucimare Ferraz

ORCID: https://orcid.org/0000-0002-2487-8614 Universidade Comunitária da Região de Chapecó, Brasil E-mail:1ferraz@unochapeco.edu.br

Kélen Antunes

ORCID: https://orcid.org/0000-0002-0380-0906 Universidade Comunitária da Região de Chapecó, Brasil E-mail: kelen.antunes@autlook.com

Junir Antonio Lutinski

ORCID: https://orcid.org/0000-0003-0149-5415 Universidade Comunitária da Região de Chapecó, Brasil E-mail: junir@unochapeco.edu.br

\begin{abstract}
Resumo
A dengue tem sido uma preocupação constante na área da saúde pelas características endêmicas e virais da doença tornando-a um problema de saúde pública. Este estudo teve como objetivo descrever indicadores para a ocorrência da dengue em municípios da região Oeste de Santa Catarina. Trata-se de uma pesquisa quantitativa de caráter descritivotransversal. A investigação ocorreu no segundo semestre de 2017 em quatro municípios (Chapecó, Descanso, Pinhalzinho e São Miguel do Oeste), que apresentaram os maiores índices de incidência de dengue no ano de 2016. Foi realizada uma busca em dados secundários na base de dados virtual do DataSus e do Instituto Brasileiro de Geografia e Estatística (IBGE) referentes aos municípios pesquisados rastreando informações sobre cobertura da Estratégia de Saúde da Família, índices de emprego e desemprego, escolaridade, Índice de Desenvolvimento Humano, saneamento básico, cobertura da rede de esgoto, gestão de resíduos, tratamento da água e taxa de urbanização. Observou-se déficits no sistema de esgotamento sanitário e percentual abaixo do esperado para a avaliação do IDEB no Ensino Médio. A urbanização acelerada favorece o aparecimento de situações que contribuem para a ocorrência de dengue. Indicadores como IDH, cobertura adequada da ESF podem ser fatores de proteção e prevenção da dengue.

Palavras-chave: Doenças endêmicas; Políticas públicas; Promoção da saúde; Vetores.
\end{abstract}

\begin{abstract}
Dengue fever has been a constant concern in the health area due to the endemic and viral characteristics of the disease, making it a public health problem. This study aimed to describe indicators for the occurrence of dengue in municipalities in the western region of Santa Catarina. This is a quantitative descriptive-cross-cutting research. The investigation took place in the second half of 2017 in four municipalities (Chapecó, Descanso, Pinhalzinho and São Miguel do Oeste), which had the highest rates of dengue incidence in 2016. A search was performed on secondary data in the virtual database of DataSus and the Brazilian Institute of Geography and Statistics (IBGE) for the municipalities surveyed tracking information on coverage of the Family Health Strategy, employment and unemployment rates, education, Human Development Index, basic sanitation, coverage of the sewage network, waste management, water treatment and urbanization rate. There were deficits in the sanitary sewage system and a percentage lower than expected for the evaluation of the IDEB in High School. Accelerated urbanization favors the emergence of situations that contribute to the occurrence of dengue. Indicators such as HDI, adequate coverage of the ESF can be protective and prevention factors for dengue.
\end{abstract}

Keywords: Endemic diseases; Public policy; Health Surveillance.

\section{Resumen}

El dengue ha sido una preocupación constante en el área de la salud por las características endémicas y virales de la enfermedad, lo que la convierte en un problema de salud pública. Este estudio tuvo como objetivo describir indicadores de ocurrencia de dengue en municipios de la región oeste de Santa Catarina. Se trata de una investigación 
cuantitativa descriptiva-transversal. La investigación se llevó a cabo en el segundo semestre de 2017 en cuatro municipios (Chapecó, Descanso, Pinhalzinho y São Miguel do Oeste), que tuvieron las mayores tasas de incidencia de dengue en 2016. Se realizó una búsqueda de datos secundarios en la base de datos virtual de DataSus y el Instituto Brasileño de Geografía y Estadística (IBGE) para los municipios encuestados rastreando información sobre cobertura de la Estrategia de Salud de la Familia, tasas de empleo y desempleo, educación, Índice de Desarrollo Humano, saneamiento básico, cobertura de redes de alcantarillado, gestión de residuos, tratamiento de agua y urbanización. Hubo déficits en el sistema de alcantarillado sanitario y un porcentaje menor al esperado para la evaluación del IDEB en Bachillerato. La urbanización acelerada favorece el surgimiento de situaciones que contribuyen a la ocurrencia del dengue. Indicadores como el IDH, la cobertura adecuada de la ESF pueden ser factores de protección y prevención del dengue.

Palabras clave: Enfermedades endémicas; Políticas públicas; Vigilancia de la salud.

\section{Introdução}

A dengue tem sido foco de preocupação para autoridades em saúde no mundo, em razão da expansão geográfica e da dificuldade para o controle desta endemia (Teixeira et al., 2015). No Brasil, as epidemias de dengue, considerando a complexidade, muitas vezes ultrapassam a capacidade de atendimento no Sistema Único de Saúde (SUS) (Teixeira et al., 2015). Formas de controle e prevenção de casos da doença são objetivos de diversas tecnologias utilizadas com essa finalidade, bem como de drogas antivirais e a criação de técnicas para o monitoramento populacional dos mosquitos vetores em suas diversas fases de vida, mesmo considerando-se situações em que os recursos destinados ao controle do vetor sejam apropriados para a implementação de programas, muitas vezes não se tem alcançado sucesso (Zara et al., 2016).

A infecção produzida pelo vírus DEN apresenta uma situação epidemiológica preocupante em vários países (WHO, 2012). A transmissão do agente etiológico tem sido registrada de uma forma cada vez mais intensa, aumentando a magnitude das epidemias e a área de circulação do vírus (Teixeira et al., 2015; Valle, Pimenta \& Cunha, 2015). Os fatores favoráveis no Brasil colocam o país no topo do ranking para ocorrência de casos de dengue. Observa-se que a doença está presente em toda a região intertropical e expandindo-se para regiões subtropicais (Valle et al.2015). Ocorre na forma epidêmica, aumentando a incidência e a prevalência de casos anualmente (Brasil, 2012). No estado de Santa Catarina, no ano de 2016, foram notificados mais de 13 mil casos suspeitos da doença com confirmação de mais de 30\% destes. Dentre os municípios do estado, no Oeste estão aqueles que lideraram o ranking estadual dos casos confirmados. Ainda no estado, em 2017, foram notificados 2.498 casos da doença, desses, 16 (1\%) foram confirmados por critério laboratorial. Comparando 2016 com 2017 , quando foram notificados 2.498 casos, observa-se uma redução de $82 \%$ (Santa Catarina, 2018).

Dentro desse contexto, o surgimento do aumento de casos de dengue pode estar associado às atividades humanas desordenadas que modificam o ambiente e as condições socioambientais ou pela ineficiência dos programas de combate ao vetor (Daher et al., 2013). Considerando-se a multiplicidade de fatores que potencializam a ocorrência de eventos de transmissão viral de dengue, tora-se relevante verificar aqueles que mais se destacam na região do estudo. Contextos econômicos, políticos e sociais influenciam as condições de vida, saúde e formas de adoecimento da população. A maior parte das doenças e suas iniquidades existentes em um país, por exemplo, as desigualdades em saúde (Pimenta, 2015) são decorrentes das condições em que as pessoas vivem, nascem e produzem (Carrapato et al., 2017). A compreensão acerca dos determinantes de saúde e seus indicadores reflete as condições em que uma determinada população vive. O estado de saúde de uma população pode ser definido pelas interações de aspectos sociais, econômicos, culturais e políticos, levando em consideração as questões de saúde de forma indissociável das questões ambientais (Carrapato et al., 2017).

Nesse sentido, dentre os determinantes de saúde para a ocorrência de dengue, Valle et al. (2015) relatam uma diversidade de fatores sociais e ambientais relacionados à ocorrência da dengue. Ao relacionar as áreas ambientais e geográficas, o crescimento populacional e a urbanização (Zara et al., 2016), relacionamos também o vetor e hospedeiro, agente e vetor, abastecimento de água, condições pluviométricas e condições socioeconômicas que tendem a influenciar na ocorrência da doença nos variados contextos espaciais e temporais (Teixeira et al., 2015). 
Desse modo, com o objetivo de reduzir a incidência da doença, as ações intersetoriais mostram-se cada vez mais necessárias para o êxito do controle das arboviroses. De acordo com o Programa Nacional de Combate à Dengue (PNCD) é necessário o monitoramento das áreas regionais com base no mapeamento de risco, para a realização de um conjunto adequado de medidas de controle vetorial. O mapeamento permite analisar de forma mais precisa as situações de risco (Silva et al., 2015; Zara et al., 2016). Dentre as formas de suporte estão os sistemas de informação estaduais, departamentos estaduais e municipais de Vigilância Epidemiológica e Ambiental que atuam no bloqueio da transmissão e no monitoramento e controle da infestação pelo vetor (Feitosa et al., 2016). E especificamente em Santa Catarina, a Diretoria de Vigilância Epidemiológica monitora e atua em conjunto com municípios na prevenção e no controle de eventos de transmissão viral da doença.

Casos de dengue têm sido registrados somente na última década do estado catarinense. Neste contexto, torna-se relevante conhecer a realidade social, cultural e econômica presento nos municípios da região, bem como indicadores que podem subsidiar as políticas públicas de saúde.

Diante desse contexto, destaca-se a importância e relevância deste trabalho, uma vez que a dengue emerge como um desafio à saúde no Brasil e no mundo, já que acomete centenas de milhares de pessoas todos os anos somente no Brasil e causa centenas de óbitos. Assim, o objetivo deste estudo foi descrever indicadores para a ocorrência da dengue em municípios da região Oeste de Santa Catarina.

\section{Metodologia}

Este estudo seguiu todas as determinações da Resolução 466/2012 da Comissão Nacional de Ética e Pesquisa com Seres Humanos (Brasil, 2012). O projeto foi encaminhado para as Secretarias Municipais de Saúde para autorização e posteriormente foi submetido ao Comitê de Ética em Pesquisa envolvendo Seres Humanos e aprovado sob Parecer $\mathrm{n}$. 2.249.830.

Trata-se de uma pesquisa quantitativa de caráter descritiva-transversal. O estudo foi desenvolvido no período de setembro a dezembro de 2017 nos municípios de Chapecó, Descanso, Pinhalzinho e São Miguel do Oeste, região Oeste de Santa Catarina, Brasil (Dyniewicz, 2014).

Em Santa Catarina, Chapecó apresenta-se como o município mais populoso da região Oeste de Santa Catarina, e a maior densidade demográfica. Por ser o maior polo econômico da região Oeste catarinense, apresenta maior renda per capita dentre os municípios estudados (Santa Catarina, 2018; Ibge, 2021).

Os municípios escolhidos para o estudo estão entre aqueles que, em 2016, se destacaram quanto às taxas de incidência de dengue no estado: Pinhalzinho com 60,9\%; Chapecó com 19,7\%; Descanso com 2,2\%; e São Miguel do Oeste com 1\% (Santa Catarina, 2018) (Figura 1). 
Figura 1 - Serviços de Saúde nos municípios de Chapecó, Descanso, Pinhalzinho e São Miguel do Oeste, 2018.

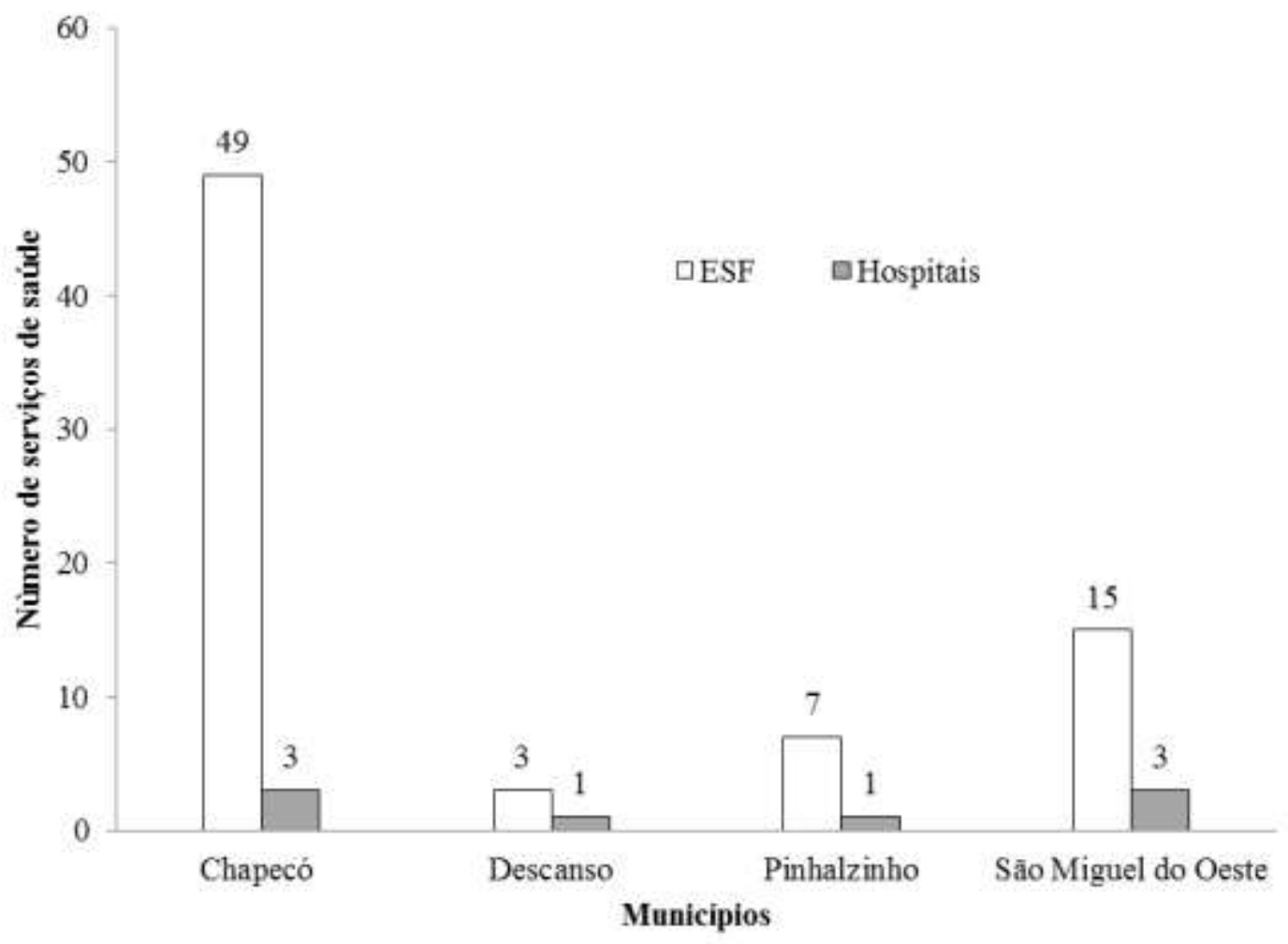

Fonte: Autores (2018).

Foi realizada uma busca em dados secundários na base de dados virtual do descrever a sigla aqui (DATASUS) e do Instituto Brasileiro de Geografia e Estatística (IBGE) referentes ao municípios pesquisados rastreando informações sobre escolaridade, índice de desenvolvimento humano (IDH), cobertura da Estratégia de Saúde da Família (ESF), índices de emprego e desemprego, escolaridade, Índice de Desenvolvimento Humano, saneamento básico, cobertura da rede de esgoto, gestão de resíduos, tratamento da água e taxa de urbanização.

Os resultados foram tabulados em um banco de dados no software Excel (versão 2010). Foram utilizadas técnicas de análise descritiva exploratória por meio de análises de frequência.

\section{Resultados}

Entre as cidades de Santa Catarina, incluídas neste estudo, quanto a comparação do Índice de Desenvolvimento Humano (IDH), São Miguel do Oeste apresentou melhor índice (Tabela 1). 
Tabela 1 - População, densidade demográfica, renda per capita e IDH dos municípios de Chapecó, Descanso, Pinhalzinho e São Miguel do Oeste, 2017.

\begin{tabular}{ccccc}
\hline Município & $\begin{array}{c}\text { Número de } \\
\text { habitantes }\end{array}$ & $\begin{array}{c}\text { Densidade } \\
\text { demográfica }\end{array}$ & $\begin{array}{c}\text { Renda per capita } \\
\text { (R\$) }\end{array}$ & IDH \\
\hline Chapecó & 213.279 & 293,1 & $37.303,1$ & 0,79 \\
Descanso & 8.399 & 30,2 & $24.368,7$ & 0,74 \\
Pinhalzinho & 19.511 & 127,4 & $47.351,8$ & 0,78 \\
São Miguel do Oeste & 39.793 & 155,1 & $33.397,2$ & 0,80 \\
\hline
\end{tabular}

Fonte: Autores IBGE (2017).

A taxa de escolarização nos municípios na faixa de seis a 14 anos está acima de 97\% em todos os municípios avaliados. As taxas de escolarização, segundo o Índice de Desenvolvimento da Educação Básica (IDEB), apresentam pouca variação entre os municípios, tanto no ensino fundamental $(\mathrm{EF})$ (Amplitude $=0,3)$, quanto no ensino médio (EM) (Amplitude $=0,6)($ Tabela 2).

Tabela 2 - Taxa de escolarização e Índice de Desenvolvimento da Educação Básica (Ensino Fundamental e Médio) dos municípios de Chapecó, Descanso, Pinhalzinho e São Miguel do Oeste, 2017.

\begin{tabular}{cccc}
\hline Município & Taxa de escolarização (6 a 14 anos) & IDEB (EF) & IDEB (EM) \\
\hline Chapecó & 98,4 & 6,4 & 5,1 \\
Descanso & 99,2 & 6,4 & 5,4 \\
Pinhalzinho & 99,2 & 6,2 & 4,9 \\
São Miguel do Oeste & 97,7 & 6,5 & 4,8 \\
\hline
\end{tabular}

Fonte: Autores. (2017), IBGE (2017).

Segundo dados do IBGE, o município de Pinhalzinho conta com 94,9\% de cobertura de esgotamento sanitário. Em contraste, o município de Descanso apresenta apenas 13,2\%. Chapecó e São Miguel do Oeste estão na faixa entre 60 e $70 \%$ (Figura 2). 
Figura 2 - Cobertura do esgotamento sanitário nos municípios de Chapecó, Descanso, Pinhalzinho e São Miguel do Oeste, 2017.

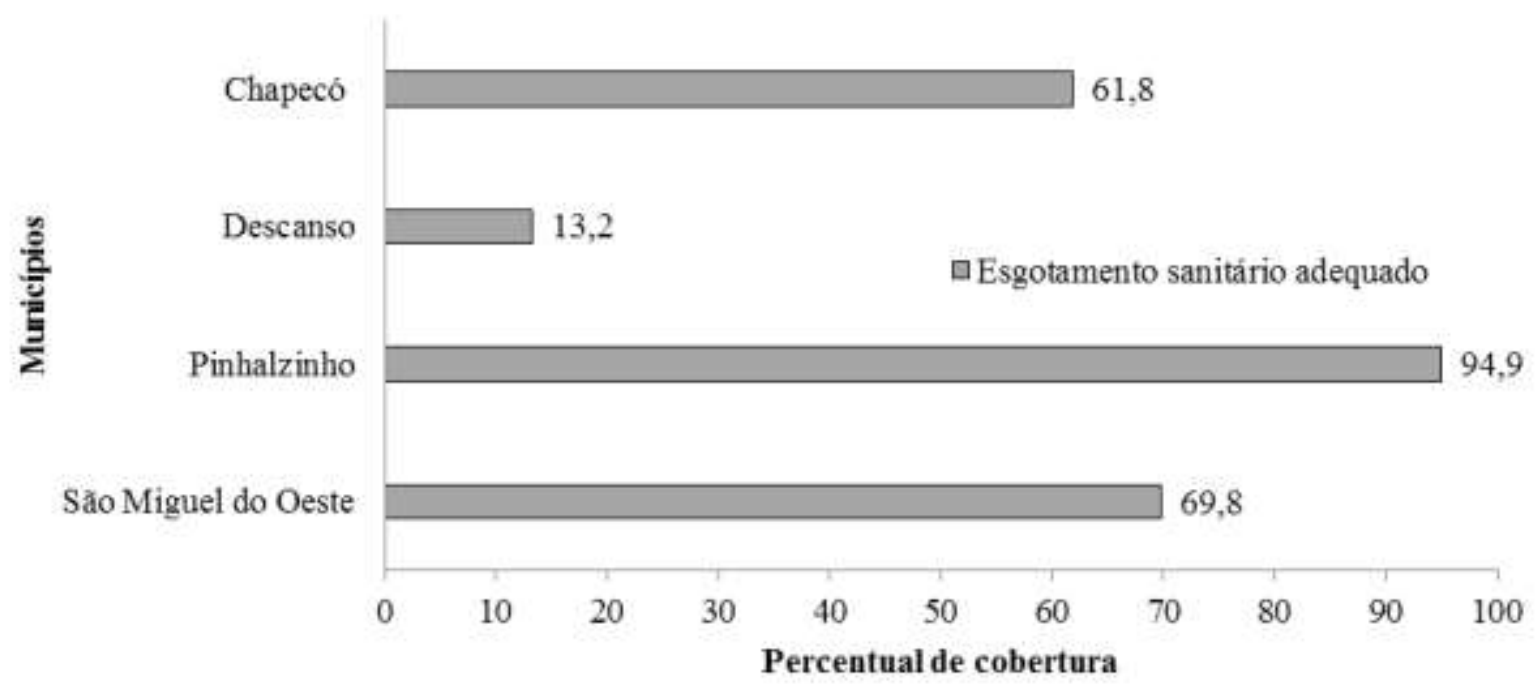

Fonte: Autores (2017), IBGE (2017).

\section{Discussão}

No presente estudo os municípios de Chapecó, Descanso e Pinhalzinho ficaramcaracterizados como municípios com IDH médio e São Miguel do Oeste com IDH alto, isso reflete positivamente e é considerado um fator de proteção no combate e prevenção da dengue, conforme estudo de Feitosa et al. (2016).. O resultado de valores obtidos do cálculo parte de zero (pior situação de desenvolvimento humano) até um (melhor situação de desenvolvimento humano). Um município ou região tem alto desenvolvimento humano se apresentar valores IDH for igual ou maior a 0,8; médio, entre 0,79 a 0,5; e baixo, se for menor ou igual a 0,49 (Cortés et al., 2015).

Os resultados desta pesquisa corroboram Araújo (2014) ao apontar que a densidade demográfica e o crescimento da população, com destaque para a população urbana de forma desordenada, aumentaram de forma considerável e tal fenômeno pode ser considerado como decisivo para epidemias da dengue. A transição demográfica da população para as cidades demanda uma maior pressão sobre os recursos naturais disponíveis em sua área de abrangência, seja por alimentos, seja por recursos energéticos ou naturais (extração de materiais para a construção civil). Municípios que sofrem mudanças no número de habitantes em um dado período podem apresentar alterações no uso do solo do seu território, tanto em áreas urbanizadas, quanto em seus arredores (Rufino \& Silva, 2017). Desta forma, esta variação do contingente populacional aumenta a demanda de recursos, a densidade demográfica e reflete o crescimento desordenado, comum em muitos centros urbanos. Como consequência, há um aumento da produção de resíduos sólidos e na demanda por água, fatores diretamente associados com a proliferação do vetor da dengue e com situações de transmissão viral da doença (Araújo, 2014).

Em relação ao resultado sobre o IDH e a escolaridade e saneamento básico, Oliveira (2018), em seu estudo indica que há desigualdades e negligência em saúde em populações empobrecidas. O baixo nível de renda gera saúde precária e essa, por sua vez, tende a causar um baixo nível de renda. A população de maior renda tende a usufruir de maior qualidade de vida e ter maior acesso a serviços de saúde, seja público, seja privado. A causalidade é relação entre um evento (uma causa) e um segundo evento (um efeito), em que o segundo acontecimento é entendida como uma consequência do primeiro (Hume, 2000).

Em relação à renda nos municípios estudados, esta pode estar relacionada com a dengue no sentido de acesso a bens e serviços de saúde, moradia e educação, levando a população a ignorar riscos de aparecimento de focos do vetor ou entender que a prevenção à dengue depende somente do poder público, não adotando ou não seguindo recomendações de prevenção. 
Uma renda maior afeta a saúde, uma vez que oportuniza melhor aproveitamento de status de saúde, permitindo o acesso aos bens e serviços de saúde, acesso de saúde, moradia e educação. A saúde pode afetar a renda e, neste sentido, a saúde é decisória na produtividade e disponibilidade para o trabalho (Oliveira, 2018).

No caso dos municípios estudados, aqueles com maior acesso da população são os serviços de saúde (Figura 1) com atendimento pelo SUS, em que cada município conta com pelo menos um serviço desta categoria. Dentro das atribuições deste serviço de atenção, conseguem atender pacientes com dengue. Os hospitais, de pequeno ou de médio porte, devem estar preparados para atender pacientes com alguma manifestação da dengue, com ou sem sinais de alarme. Crianças, idosos, gestantes ou pacientes com alguma comorbidade, que necessitam de hidratação oral ou venosa, supervisionada pela equipe de enfermagem e com avaliação médica contínua, necessitam de assistência oportuna provida de recursos humanos e materiais de qualidade (Fausto et al., 2014).

Independentemente da classificação jurídica administrativa ou de gestão, o custeio de ações e serviços prestados com vínculo ao SUS é pactuado por contratos pré-estabelecidos entre os órgãos prestadores de serviço e gestão pública, com metas firmadas para serem atingidas pela instituição de saúde e definindo valores que serão repassados com finalidade de financiar os gastos e investimentos em saúde (Holz et al., 2016). O financiamento não se constituiu em um limitador para as ações de controle da dengue nos municípios avaliados, considerando-se que todos tiveram sucesso na contenção da transmissão viral.

No que se refere aos indicadores de escolarização, o Índice de Desenvolvimento da Educação Básica (IDEB) tem sido um instrumento de avaliação para produzir informações da realidade educacional, com intensão de contribuir para a melhoria da qualidade da educação brasileira baseada na taxa de aprovação escolar e na média de desempenho nos exames aplicados pelo Instituto Nacional de Estudos e Pesquisas Educacionais Anísio Teixeira (INEP). Parte de três conceitos: aprovação escolar, evasão escolar e desempenho nas avaliações de língua portuguesa e matemática aplicada pela Prova Brasil com pontuação que varia de zero a dez (Barbosa \& Mello, 2015). Para o Ministério de Educação e Cultura (MEC), até 2022, o Plano de Desenvolvimento da Educação tem como meta a média seis que corresponde a um sistema educacional de qualidade quando comparado a países desenvolvidos. O que se percebe, segundo os dados dos municípios, são as notas das avaliações condizentes com o esperado pelo MEC para o ensino fundamental e abaixo do esperado para o ensino médio. A escolaridade, renda e IDH dos municípios avaliados contribuem positivamente para a prevenção à dengue, já que os valores encontrados estão, em sua maioria, acima da média nacional.

O êxodo rural concentrou a população nas cidades. Pressionadas pela demanda, estas, acabaram por não oferecer condições adequadas de habitação e saneamento básico (esgotamento sanitário, coleta de resíduos e abastecimento de água) para as parcelas da população (Teixeira et al., 2014). Verificam-se fragilidades nos municípios estudados quanto ao saneamento básico. Embora se perceba cobertura na gestão de resíduos sólidos, o abastecimento de água e o tratamento de efluentes não cobre todas as áreas urbanas e rurais. A partir dos resultados, verificam-se fragilidades quanto à prevenção à dengue, especialmente no que tange a gestão dos Resíduos sólidos Urbanos.

Teixeira et al. (2014), em sua pesquisa sobre a consequências da deficiência ou falta de saneamento básico no Brasil, no período de 2001 a 2009, relatam a ocorrência de quatro doenças com maior número de casos notificados naquele período que foram dengue, hepatite, esquistossomose e leptospirose. A prestação deficitária de serviços públicos de saneamento básico resulta em um quadro de doenças associadas ao saneamento básico desproporcional ao grau de desenvolvimento esperado. Assim, doenças infecciosas como a dengue continuam sendo causas de morbidade e mortalidade associada a fragilidades no sistema de infraestrutura sanitária (Teixeira et al., 2014). A cobertura de rede de esgotamento sanitário representa outra fragilidade nos municípios avaliados. Embora tenha-se verificado avanços neste aspecto, a maior parte da população não conta com a rede pública de coleta e tratamento de efluentes o que representa não apenas um fator associado à dengue, mas também com outras doenças veiculadas pela água não tratada. 


\section{Conclusão}

Nos municípios avaliados observa-se a ocorrência de deficits no saneamento básico e percentual abaixo do esperado para a avaliação do IDEB no ensino médio e ainda, urbanização acelerada que podem favorecer o aparecimento de situações que contribuem para a ocorrência de dengue.

Indicadores como IDH, cobertura adequada da ESF podem ser fatores de proteção e prevenção da dengue. O conhecimento dos Determinantes Sociais da Saúde oferece melhor compreensão da realidade local e gera opções de enfrentamento para desenvolver estratégias e ações de superação para o controle e combate à dengue, conforme as especificidades da região.

\section{Agradecimentos}

À Universidade Comunitária da Região de Chapecó, pelo apoio à pesquisa e à produção científica.

\section{Referências}

Araújo, R. R. (2014). Clima e vulnerabilidade socioespacial: uma avaliação dos fatores de risco na população urbana do município de São Luís (MA). 2014. xviii, 289 f. Tese (doutorado) - Universidade Estadual Paulista, Faculdade de Ciências e Tecnologia.

Barbosa, J. M. S., \& Mello, R. M. A. V. de. (2015). O IDEB como instrumento de avaliação da aprendizagem escolar: uma visão crítica. Eletrônica Pesquiseduca. 7(13), 106-123.

Brasil. (2012). Secretaria de Atenção à Saúde. Departamento de Atenção Básica. Política Nacional de Atenção Básica. Ministério da Saúde.

Carrapato, P., Correia, P. \& Garcia, B. (2017). Determinantes da saúde no Brasil: a procura da equidade na saúde. Saúde \& Sociedade. $26(3)$, 676-689.

Cortés, J. J. C., Honório, N. A., Gibson, G., \& Peiter, P. C. (2015). Determinantes sociais da distribuição espacial dos casos de dengue na faixa fronteiriça do Brasil. Espaço \& Geografia. 18(3), 611-638.

Daher, M. J. E., Barreto, B. T. do B., \& Carvalho, S. C. (2013). Dengue: aplicação do protocolo de atendimento pelos enfermeiros. Revista de Enfermagem da UFSM. 3(3), 440-448.

Dyniewicz, A. M. (2014). Metodologia de pesquisa em saúde para iniciantes. (3a ed.), Difusão.

Fausto, M. C. R., Giovanella, L., Mendonça, M. H. M. de, Seidl, H., \& Gagno, J. (2014). A posição da Estratégia Saúde da Família na rede de atenção à saúde na perspectiva das equipes e usuários participantes do PMAQ-AB. Saúde em Debate. 38, 13-33.

Feitosa, F. R. S., Sobral, I. S., \& Jesus, E. N. de. (2015). Indicadores socioambientais como subsídio à prevenção e controle da dengue. Revista Eletrônica em Gestão, Educação e Tecnologia Ambiental. 19(3), 351-368.

Feitosa, F. R. S., Sobral, I. S., Silva, M. do S. F. da, \& Jesus, E. N. de. (2016). Estratégias de prevenção e controle da dengue em Aracaju: potencialidades e fragilidades. Caminhos da Geografia. 17(60), 149-168.

Holz, C. B., Menezes, L. P., Begnini, D., \& Sarturi, F. (2016). O hospital na rede de Atenção à Saúde: uma reflexão teórica. Espaço Ciência \& Saúde. 4, 101115 .

Hume, D. (2000). Tratado da natureza humana. (2a ed.), Editora Unesp.

Oliveira, R. G. de. (2018). Sentidos das doenças negligenciadas na agenda da saúde global: o lugar de populações e território. Ciência \& Saúde Coletiva. 23(7), 2291-2302.

Pimenta, D. N. (2015). Determinação Social, Determinantes Sociais de Saúde e a Dengue: Caminhos possíveis? In: Valle, D., Pimenta, D. N., \& Cunha, R. V. da (Org.). Dengue: teorias e práticas. Rio de Janeiro: Fiocruz, p. 408-447.

Rufino, I. A. A., \& Silva, S. T. da. (2017). Análise das relações entre dinâmica populacional, clima e vetores de mudança no semiárido brasileiro: uma abordagem metodológica. Boletim de Ciências Geodésicas. 23(1), 166-181.

Santa Catarina. (2018). Secretaria de Estado da Saúde. Diretoria de Vigilância Epidemiológica. 26º Boletim Epidemiológico/2017. Situação da dengue, febre do chikungunya e zika vírus em Santa Catarina. Florianópolis: Secretaria de Estado da Saúde.

Santos, M. (2004). A Natureza do Espaço: técnica, razão e emoção. (4a ed.), Editora da Universidade de São Paulo.

Silva, I. B. da, Mallmann, D. G., \& Vasconcelos, E. M. R. de. (2015). Estratégias de combate à dengue através da educação em saúde: uma revisão integrativa. Saúde, Santa Maria. 41(2), 27-34. 
Research, Society and Development, v. 10, n. 10, e36101018488, 2021

(CC BY 4.0) | ISSN 2525-3409 | DOI: http://dx.doi.org/10.33448/rsd-v10i10.18488

Teixeira, J. C., Oliveira, G. S. de, Viali, A. de M., \& Muniz, S. S. (2014). Estudo do impacto das deficiências de saneamento básico sobre a saúde pública no Brasil no período de 2001 a 2009. Engenharia Sanitária e Ambiental. 19(1), 87-96.

Teixeira, M. G., Costa, M. da C. N., Barreto, M. L., \& Barreto, F. R. (2015). Epidemiologia da Dengue. In:_Valle, D., Pimenta, D. N., \& Cunha, R. V. da (Org.). Dengue: teorias e práticas. Fiocruz, p. 293-315.

Valle, D., Pimenta, D. N., \& Cunha, R. V. da (Org.). (2015). Dengue: teorias e práticas. Fiocruz.

WHO. (2012). Handbook for Clinical Management of Dengue. WHO.

Zara, A. L. de S. A., Santos, S. M. dos, Fernandes-Oliveira, E. S., Carvalho, R. G., \& Coelho, G. E. (2016). Estratégias de controle do Aedes aegypti: uma revisão. Epidemiologia e Serviços de Saúde. 25(2), 391-404. 